\title{
La construcción política del deporte en el sistema educativo peruano: una mirada a los contenidos del curso de Educación Física durante el siglo XX
}

Mariana Marceillac*

* Licenciada en Sociología y diplomada en Gestión de la Formación y Capacitación por la Pontificia Universidad Católica del Perú (PUCP). Tiene un MBA por la Escuela de Negocios de la Universidad Erasmo de Róterdam (RSM). Correo electrónico: mmarceillac@pucp.pe.

Fecha de recepción: 20/04/20. Fecha de aceptación: 08/10/20. 
La construcción política del deporte en el sistema educativo peruano: una mirada a los contenidos del curso de Educación Física durante el siglo XX

\title{
RESUMEN
}

La gestión de Educación Física en el currículo nacional expresa proyectos políticos e ideas cambiantes sobre lo que queremos que sea el deporte para las nuevas generaciones a lo largo del tiempo. Esta investigación busca identificar la utilidad sociopolítica dada por el Estado a la Educación Física a lo largo del siglo XX, identificando tres visiones distintas de deporte para la sociedad. La materia se insertó en la escuela peruana a principios del siglo XX para resolver problemáticas sociales de aquel entonces: fortalecer la raza, combatir malos hábitos y lograr la industrialización y la modernización del país. Entre 1950 y 1979, quedó íntimamente relacionada con la Educación Premilitar y la generación de una identidad nacional. Desde 1980, la reconceptualización del curso en función al desarrollo del individuo y no a intereses sociopolíticos terminaron dejando la Educación Física y el deporte al final de la cola respecto de otras prioridades del Estado. Esfuerzos por desarrollar el deporte peruano se han retomado, pero para que tengan éxito será necesario definir una visión y proyecto nacional claros, además de tomar acción desde la escuela.

Palabras clave: Educación física, deporte, currículo nacional, sistema educativo peruano

The political construction of sport in the Peruvian educational system: a look at the contents of the Physical Education course during the 20th century

\begin{abstract}
The Physical Education contents in the national curriculum express political projects and changing ideas about what we want sport to be for new generations over time. This research identifies the socio-political utility given by the Peruvian State to Physical Education throughout the 20th century, distinguishing three different visions of sport for society. The course was inserted in schools at the beginning of the century to solve social problems: strengthening the race, fighting bad habits, and achieving national industrialization and modernization. Between 1950 and 1979, it was closely related to Premilitary Education and the generation of patriotism. Since 1980, the reconceptualization of the course based on individual development and not on socio-political interests left Physical Education and sport at the end of the queue compared to other State priorities. Efforts to develop Peruvian sport have been reinitiated, but their success depends on defining a clear national vision and project, as well as taking action from schools.
\end{abstract}

Keywords: Physical education, sport, national curriculum, Peruvian educational system 


\section{INTRODUCCIÓN}

La Educación Física ha tenido diferentes roles a lo largo de la historia del sistema educativo peruano. Pasó de tener una presencia importante a inicios del siglo pasado, a la casi desaparición en la década de 1980. Cuando fue introducida en el currículo nacional en 1896 durante el gobierno de Nicolás de Piérola, el curso constituía una pieza fundamental de las políticas del Estado. Décadas después, perdió su centralidad para transformarse en un curso complementario a la Educación Premilitar y en una herramienta para formar un cuerpo de individuos disciplinados con una identidad nacional y patriótica, de gran funcionalidad para la racha de gobiernos militares que presidió al Perú entre 1950 y 1980, con excepción de los gobiernos de Manuel Prado Ugarteche y Fernando Belaunde Terry.

Finalmente, con el retorno de la democracia en 1980, la Educación Física pasó al último plano dentro del currículo escolar. No solo se la consideró en la mayoría de gobiernos como un curso de segundo orden frente a las asignaturas académicas, sino que ni siquiera las dos horas semanales que se le asignaron pudieron realizarse efectivamente ante las grandes deficiencias en el presupuesto, infraestructura y cuerpo docente, entre otros motivos. Desde entonces, la materia cayó en una situación de desatención ${ }^{1}$.

La forma como se ha implementado y proyectado la Educación Física en el currículo nacional ha ido cambiando. Para entender dichos cambios es preciso entender que los currículos nacionales expresan ideas sobre la sociedad que los gobiernos aspiran transmitir a las nuevas generaciones. Cada contenido curricular en un sistema educativo está pensado, en realidad, para alcanzar propósitos básicos de la educación y la sociedad que se anhela. Desde esa perspectiva, el que la Educación Física sea o no parte del currículo nacional expresa ideas cambiantes sobre lo que se aspira socialmente sea el deporte para las nuevas generaciones.

La presente investigación buscó mostrar cómo la importancia de la Educación Física en el currículo nacional ha variado en el tiempo, como consecuencia de los cambios en su funcionalidad política y social. Se analizó cómo los contenidos del curso se han construido social y políticamente a lo largo de su historia, mostrando los distintos roles que esta asignatura cumplió en diferentes períodos entre los años 1900 y 1990.

Tal como se abordará en las conclusiones generales, la falta de atención o interés por el curso es algo que parece estar cambiando en la actualidad. Entender lo sucedido entre 1900 y 1990 es importante para definir el trabajo futuro. 


\section{Puntos de PaRTida Desde la teoría}

La sociología del deporte constituye un primer marco de referencia para este estudio. Dos de sus principales autores estudiaron el surgimiento del deporte en la Inglaterra del siglo XVII y mostraron este fenómeno como parte del proceso civilizatorio de las sociedades (Elias y Dunning, 1992). El surgimiento del deporte moderno fue resultado del ascendente control social del Estado sobre la violencia física y la agresión, en conjugación con la disminución de la sensación de placer obtenida de la participación en actos violentos.

El deporte moderno permitió producir un equilibrio entre demasiada tensión —antes liberada por la violencia física- y el aburrimiento. También permitió sustituir impulsos y actos, como el sexo y la guerra, por actividades percibidas como mejores (Elias y Dunning, 1992, p. 77). Visto así, el deporte se constituyó como pieza fundamental en la modelación de las conciencias de los individuos y del proceso de modelación social en su conjunto; se convirtió en parte del vigoroso empuje civilizador de la sociedad (Elias y Dunning, 1992, p. 63).

Pero la importancia y la utilidad otorgadas al deporte fueron descritas con un valor relativo y cambiante, dependiente de la forma en la que actores como el Estado decidieran vincularse con él (Elias y Dunning, 1992, p. 22)2.

Por otro lado, teóricos de la educación y currículo ayudan a conocer más sobre las lógicas de funcionamiento de los sistemas educativos y su forma de reglamentar los contenidos que fabricará la escuela. La selección y elaboración de contenidos concreta oficialmente el vínculo entre la funcionalidad sociopolítica de materias como la Educación Física y los intereses de un gobierno (Dubet y Martuccelli, 1997), por lo que «...hay que tener una buena noción sobre quién controla el currículum y qué grupos de interés tienen voz» (Goodson, 2000, p. 43). El trabajo realizado buscó encontrar los intereses materiales e ideológicos que los distintos gobiernos del Perú ocultaron detrás de la inserción de la Educación Física en la educación pública.

Aunque la sociología del deporte y estudios teóricos sobre educación y currículo permitieron proponer un acercamiento inicial a este tema de investigación, su futuro desarrollo deberá ser complementado con estudios sobre el rol del ejercicio físico en las sociedades que han empezado a desarrollarse recientemente.

\footnotetext{
2 El razonamiento dado por ambos autores está intrínsecamente vinculado con el concepto de figuración, de Elias. Las figuraciones y el entramado social que las conformen quedan determinadas fundamentalmente por el nivel de poder de los actores; y en su capacidad para condicionar la situación política, económica y social de un grupo humano en cierto momento histórico. Esta investigación se enfocó en el actor de mayor poder dentro de la figuración que conforma el sistema educativo peruano, ya que es el Estado quien determina qué contenidos enseñar al estudiante con miras a formar individuos con cierta cantidad de conocimientos, actitudes y disposiciones.
} 


\section{Metodología y ALCANCES DE LA INVESTIGACión}

La metodología utilizada en esta investigación fue de corte cualitativo y constó principalmente del análisis de textos desde un enfoque histórico. Dicho proceso se trabajó en tres fases.

La primera fue una delimitación temporal que buscó identificar momentos históricos dentro del periodo de noventa años con características similares; la segunda, una revisión de fuentes primarias de los diferentes momentos identificados en la fase anterior, analizando la producción de la Educación Física y del deporte en la escuela en cada uno. Finalmente, una entrevista no estructurada constituyó la tercera y última fase del proyecto, destinada a resolver vacíos y contradicciones encontradas durante el análisis de fuentes.

Se buscó ejercer una sociología histórica, capaz de explicar la realidad de la Educación Física en base al proceso de estructuración social a lo largo del tiempo. Eso sí, puesto que la Educación Física está sujeta a proyectos educativos, sujetos a su vez a políticas educativas, sujetas a su vez al gobierno de turno, la investigación se concentró en la producción de la Educación Física y del deporte desde el Estado y se ocupó solo de analizar los cambios históricos del curso dentro del currículum escrito u oficial, mas no necesariamente el currículo implementado. Esto implicó prestar atención tanto a las estrategias discursivas como a las argumentativas utilizadas en cada documento analizado ${ }^{3}$.

La sociología histórica de Abrams, quien propone estudiar los fenómenos de transición social a través de la comprensión de las mentalidades de las sociedades pasadas (Abrams, 1983, p. ix), fue importantísima para el propósito de este trabajo: descubrir bajo qué mentalidades, creencias o racionalidades se fue definiendo y moldeando la Educación Física en el currículo nacional, y rastrear la trayectoria de la Educación Física en el currículo nacional como punto de partida para empezar a entender su realidad actual.

\section{1900-1949: LA EdUCACIÓN Física COMO PILAR dE LA MODERNIZACIÓN}

Desde los primeros años del siglo XX hasta la década de 1940, el Perú presenció la inserción progresiva de la Educación Física en las políticas educativas del Estado (Muñoz, 2001). En el marco de un discurso positivista, documentación oficial y académica de 1900-1949 muestra que la Educación Física tuvo un papel central

\footnotetext{
3 Las estrategias discursivas, por un lado, hacen referencia a las formas en las que la Educación Física fue gradualmente construida en el discurso de estos textos. Las estrategias argumentativas, por otro, a los presupuestos y explicaciones utilizadas para justificar la representación o función que se le dio a la Educación Física (Soler, 2011, p. 79).
} 
para resolver problemáticas físicas, higiénicas y morales de la sociedad peruana que eran constantemente discutidas en aquella época.

Por un lado, fue vista como vehículo para fortalecer la salud de la raza india y mestiza que identificaba al peruano como frágil. Por otro, como forma de desarrollar buenos hábitos higiénicos, incentivar las sanas actividades e imponer disciplina en la sociedad. La introducción de la Educación Física en el currículo nacional buscó en estos años generar un capital humano capaz de generar industria en el Perú y llevar al país a la modernidad.

La inserción de la Educación Física en las escuelas buscó la eugenesia: «un proceso destinado a ayudar a la nación peruana a elevarse a través de la mejora de los estándares de salud y nutrición, y mejorando la educación de los grupos considerados inferiores» (Oliart, 2011, p. 27). Este movimiento, que fue promovido activamente por la élite aristocrática desde inicios del siglo XX, trascendió el ámbito escolar y fue parte de un proyecto mayor que buscó la masificación del deporte, la erradicación de malos hábitos enraizados en la sociedad y la modernización del país (Muñoz, 2001). De ahí que haya tomado años en desarrollarse y que se haya mantenido vigente durante medio siglo.

\subsection{Funciones adjudicadas a la educación física entre 1900 y 1949}

La Educación Física fue un punto de partida estratégico para el gran proyecto de modernización iniciado durante la República Aristocrática en busca de individuos eficientes para la constitución de un Estado moderno. Este proyecto encontró sustento en dos hechos de la historia.

Primero, la derrota sufrida en la Guerra del Pacífico que puso énfasis en la necesidad de fortalecer la raza y revitalizar la moral del combatiente peruano. Eso explica que las primeras medidas tomadas para implementar la Educación Física en las escuelas hayan sido dictaminadas en el gobierno de Nicolás de Piérola, en 1896, tras la guerra. Piérola reglamentó la instrucción física y la educación moral en las escuelas, aunque la implementación de la gimnasia y los ejercicios militares se dio en 1910 (Muñoz, 2001, p. 258-269).

Segundo, la expansión de malos hábitos como el juego y el consumo de alcohol, que alcanzaron un nivel crítico y obligaron al Estado a tomar políticas para sancionarlos y reemplazarlos por actividades moral y saludablemente convenientes. Existió una jerarquización de las razas según la cual los indígenas, criollos y negros eran vistos, en palabras de la época, como «... faltos de ideales decisivos, incapaces hasta ahora de elaborar su propia suerte» (Eguiguren, 1915, p. 8). La apuesta era forjar un hombre cabal y civilizado, «hecho y derecho», y la escuela se convirtió en la responsable de desarrollar el carácter y la voluntad de estos hombres, capacitando a sus alumnos para el trabajo industrial y las actividades en sociedad civilizada. 
La inserción de la Educación Física en las escuelas buscó, por un lado, formar a un hombre viril y sano en favor de una "eugenesia colectiva». Estas ideas se ven reflejadas en los escritos de grandes pensadores de la época:

Considerando de este modo la vida, como fuerza física y biológica, puesta al servicio de la dinámica espiritual, es un valor que debe tener en cuenta la técnica de la educación, para aumentar su eficiencia y eliminar los fenómenos que se opongan a su desarrollo [...] De ahí la importancia de los ejercicios gimnásticos, capaces, al mismo tiempo, de vigorizar sus miembros, de evitar su decadencia y de ofrecer a las aspiraciones del espíritu los medios que este necesita para mantener en progreso constante... (Deustua, 1902, p. 231).

Por otro lado, hubo también una intención de generar buenos hábitos y disciplina en la sociedad. La Educación Física también fue carta importante para eliminar las malas costumbres y reemplazarlas por actividades deseables. Por eso, muchos de los planes educativos entre 1900 y 1949 incluyeron como parte del curso de Educación Física otros contenidos de aprendizaje, más allá de los ejercicios físicos en sí mismos. Así sucedió con la Ley Orgánica de Instrucción (Gobierno del Perú, 1901), que estableció los ejercicios físicos como obligatorios en la escuela primaria y secundaria.

Sobre dicha legislación se elaboró el Plan de Educación para la escuela primaria (Gobierno del Perú, 1906), que especificó los títulos y contenidos básicos que, por ańos lectivos, debían desarrollarse en la escuela estatal. En el ámbito de la Educación Física, el documento estableció no solo ejercicios de flexibilidad, series de saltos, carreras, danza, natación o gimnasia como contenidos a desarrollarse. Dentro de la misma asignatura se incluyeron también ejercicios de orden, marchas, cantos patrióticos e incluso el desarrollo de nociones de higiene para ser trabajados con los alumnos entre el primer y el quinto año de primaria.

Posteriormente, el Reglamento General de Instrucción Media, Especial, Academias y Cursos Libres (Ministerio de Instrucción, 1912) prohibió a los alumnos a portar dinero, bebidas alcohólicas o instrumentos punzocortantes, y restringió la celebración de ventas, rifas o juegos similares. A su vez, exigió a los alumnos a presentar durante su matrícula artículos de aseo personal. como cepillo de dientes, cepillo de ropa, cepillo de cabeza, cepillo para zapatos, camisas de día, camisas de noche, pares de medias, ropa interior, pañuelos, etc.

Años después, la Ley Orgánica de Educación Pública n. 9359 (Gobierno del Perú, 1941) creó en la Dirección de Educación Física e Higiene Escolar y le asignó responsabilidades como reglamentar el servicio médico escolar, supervisar la educación sexual, asegurar el respeto a las prescripciones higiénicas y organizar la extensión de la higiene dentro y fuera de la escuela. 
Adicionalmente a las cuestiones eugenésicas, conductuales y de higiene que la República Aristocrática promovió durante las primeras décadas del siglo XX, documentos como el Reglamento General de Instrucción Media, Especial, Academias y Cursos Libres o la Guía y Reglamento General de Educación Física (Ministerio de Instrucción, 1912, 1920) vincularon estrechamente los ejercicios físicos a los ejercicios militares. Se los declaró obligatorios en todos los establecimientos de enseñanza, y su estrecho vínculo se evidenció en el carácter militarizado de las actividades físicas que describen estos textos: posiciones como las de atención y descanso, ejercicios de orden, formaciones, marchas y voces de mando son algunos ejemplos.

Importante es notar que ambos documentos fueron publicados con Augusto B. Leguía al poder: el primero durante su primer gobierno, y el segundo, a inicios del Oncenio. En este sentido, la estrecha vinculación de la Educación Física con la Educación Premilitar parecen ser un distintivo de sus políticas, más que una asociación promovida por la élite aristocrática de los primeros años.

Todo esto se condensó también en el Plan de Estudios y Programas para las Escuelas Fiscales de Enseñanza Primaria conforme al Plan Oficial (Ministerio de Educación Pública, 1937) durante el segundo gobierno del general Óscar R. Benavides, que incorporó los ejercicios físicos y las actividades militares de manera gradual para todos los niveles educativos. Esto condensa la gran amplitud con la que se concibió la Educación Física, a partir de los grandes objetivos que el Estado pretendió alcanzar con la asignatura: cuerpo y conducta.

Pero como ya se dijo, la inserción de la Educación Física en el currículo nacional no fue solo un proyecto educativo, sino uno de mayor envergadura. Al fortalecer los cuerpos de la sociedad peruana y enderezar sus hábitos, los gobiernos entre 1900 y 1949 buscaban formar mano de obra capaz de empujar la industrialización y modernización del país:

Como dice un pensador contemporáneo, "En las luchas industriales la victoria vá siempre unida al vigor físico de los productos». La educación física es la mejor escuela del carácter y de la iniciativa individual; el ejercicio es acción y movimiento. Los ingleses estudian menos que nosotros, se dice, pero son más capaces de obrar. Todos los que se han ocupado de estudiar «el porqué de ese vital genio emprendedor de los ingleses, holandeses, dinamarqueses, que tanta prosperidad han llevado a sus países y a sus individuos, están acordes en declarar, que esto se debe a un principio de educación distinto del que siguen los países latinos; lo atribuyen a la práctica constante del sport, al hábito perenne de lucha, desde la primera edad hasta los albores de la vejez. Estos continuos ejercicios atemperan las inteligencias, acostumbran al individuo a utilizar sus propias energías y valerse de si mismos para afrontar lo desconocido» (Pinto, 1915, p. 8). 
La cita anterior explica por qué el Reglamento General de Instrucción Media, Especial, Academias y Cursos Libres (Ministerio de Instrucción, 1912) justificó la inserción de la Educación Física y la Educación Premilitar apelando a los efectos económicos de su aprendizaje, y por qué, al desarrollar los contenidos del modelo de lección de Educación Física, la Guía y Reglamento General de Educación Física (Ministerio de Instrucción, 1920) asoció muchos de los ejercicios físicos al trabajo futuro de los educandos.

Aunque no a simple vista, estos gobiernos consiguieron hallar un lazo entre Educación Física y el proceso de modernización: «... el Estado y la élite comprendieron la utilidad del deporte en la formación del hombre viril, con voluntad y capacidad de acción que el Perú necesitaba» (Muñoz, 2001, p. 252). El deporte y el curso se convirtieron en un instrumento clave del Estado para ejecutar el proyecto modernizador, una propuesta educativa, política y social, con miras a construir y fortalecer la patria.

Cumpliendo un papel protagónico dentro de la coyuntura, los contenidos de Educación Física se convirtieron en los más desarrollados dentro de documentos como el Plan de Educación, el Reglamento General de Instrucción Media, Especial, Academias y Cursos Libres, la Guía y Reglamento General de Educación Física, el Plan de Estudios y Programas para las Escuelas Fiscales de Enseñanza Primaria conforme al Plan Oficial y la Ley Orgánica de Educación Pública n. 9359 (Gobierno del Perú, 1906, 1941; Ministerio de Instrucción, 1912, 1920; Ministerio de Educación Pública, 1937).

Los documentos mencionados revelan una concepción de Educación Física amplia, en donde la materia tenía como función la configuración de cuerpos en forma; la promoción de hábitos de higiene adecuados, el fomento de comportamientos pulcros y correctos y la preparación de los alumnos para el trabajo duro por el desarrollo del país.

\subsection{Alcances en la práctica entre 1900 y 1949}

Un primer aspecto en donde la Educación Física cobró importancia y notoriedad fue en la distribución de horas lectivas por curso. En el Plan de Estudios y Programas para los Colegios de Instrucción Media (Ministerio de Instrucción, 1929) estableció tanto el número de horas como los contenidos de cada curso dentro de la educación media, adjudicando a la Educación Física tres horas semanales durante el primer, segundo, quinto y sexto año de secundaria. Se le destinó la misma cantidad de horas que a materias como Geografía, Historia Antigua o Lenguas Vivas, y mayor cantidad de horas semanales que al curso de Historia del Perú. Ello sugiere una poca intención de reavivar el pasado nacional y una clara apuesta por formar individuos física y mentalmente, con vigor y disciplina. 
Adicionalmente, este documento destinó cuatro horas adicionales de Educación Militar para los alumnos de sexto año, elevando la importancia de la buena condición física y de la correcta disciplina por sobre conocimientos académicos comúnmente valorados.

Esta distribución horaria se repetiría en otros documentos, como el Decreto Supremo 39 (Ministerio de Educación Pública, 1939), que priorizó la carga horaria semanal de la Educación Física y la Instrucción Militar sobre otro tipo de asignaturas de carácter académico: Historia General, Historia del Perú o Educación Moral y Religiosa.

Un segundo aspecto que evidenció el impulso dado por el Estado y la élite al desarrollo de la Educación Física fue el institucional. La inserción de la Educación Física como curso fundamental en el currículo conllevó a la creación de numerosas instituciones para gestionar, supervisar, desarrollar y promover de la mejor manera la actividad física en los colegios.

Desde inicios de siglo, las iniciativas por insertar la Educación Física en las escuelas buscaron apoyo y asesoría extranjera, ahí en donde el deporte estaba más desarrollado. Los primeros especialistas en Educación Física que vinieron al Perú fueron parte de una misión militar francesa y llegaron a bordo del buque alemán Tanis, en 1903 (Cajas, 1957, p. 88). Consigo, trajeron la metodología de la escuela francesa de Joinville le Pont, cuya importancia y protagonismo alcanzaron su mayor auge luego de la Primera Guerra Mundial.

Aunque la misión francesa mencionada trajo consigo conocimientos sobre la gimnasia militar francesa, aportó poco en la organización del cuerpo docente: «... el error de no fundar una escuela para la preparación del profesorado. Se dictaron cursillos, pero no se pudo formar conciencia magisterial» (Cajas, 1957, p. 87).

Lo mismo sucedió con las misiones estadounidenses que llegaron después, que importaron los juegos deportivos populares en dicho país y su respectiva reglamentación, pero que no constituyeron ningún aporte en materia de formación del profesorado (Cajas, 1957, p.110).

$\mathrm{Al}$ final de las visitas francesa y estadounidenses, lo que se daba en las escuelas eran importaciones de metodologías y reglamentaciones, sin un personal capacitado para su supervisión y enseñanza (Pinto, 1915, p. 43).

De ahí que la fundación de la Escuela Nacional de Educación Física ${ }^{4}$, la creación de la Dirección Nacional de Educación Física como dependencia del Ministerio de Instrucción y la oficialización de los primeros programas de Educación Física en las

\footnotetext{
4 Antes de la creación de la Escuela Nacional de Educación Física en 1931, «... los que se dedicaban a esta enseñanza o eran maestros primarios que poseían algunas cortísimas orientaciones técnicas o eran monitores de gimnasia preparados en la Escuela Militar de Chorrillos, en rápidos cursillos, o eran exatletas o deportistas aficionados» (Belevan-García, 1962, p. 140).
} 
escuelas públicas hayan constituido un hito en la historia de la Educación Física en el Perú (Agustín, 1934).

Otros avances de la Educación Física en el ámbito institucional vinieron con la llegada de la nueva Ley Orgánica de Educación Pública (ley 9359) (Gobierno del Perú, 1941), que formó la Dirección de Educación Física e Higiene Escolar dentro del Ministerio de Educación Pública. A esta dirección se le responsabilizó de tareas como la adopción de escalas de calificación adecuadas a nuestras condiciones étnicas para medir el desarrollo de los alumnos; la vigilancia del estado físico y mental de los alumnos; la supervisión de la educación sexual en los estudiantes; la regulación de la higiene tanto dentro como fuera de las escuelas, y la dirección y regulación de la enseñanza de Educación Física. La variedad de funciones abarcadas por esta dirección muestra de nuevo el carácter amplio con el que se concibió la Educación Física en un primer momento.

Por último, esta ley creó los patronatos escolares como un organismo de cooperación entre las escuelas y la comunidad. Los patronatos debían promover la Educación Física a través de la creación de campos deportivos y parques infantiles; y difundir un espíritu patriótico entre los niños que estimulase la educación física y premilitar, el culto a los héroes y el sentimiento de honor nacional.

\section{1950-1979: EdUCACIÓN FísiCA COMO CUNA DEL PATRIOTISMO}

Los años 1950-1979 se caracterizaron por una expansión territorial del servicio educativo en desmedro de su calidad. Durante los años las décadas de 1950 a 1970 hubo una serie de proyectos estatales para ampliar el sistema educativo. Pero con todos los esfuerzos enfocados en alzar la cobertura educativa en el ámbito rural y satisfacer así la demanda de grupos desfavorecidos, se inició algo que hoy denominamos el «mito de la educación»: una búsqueda de desarrollo por medio de la escuela que en la práctica radicó más en expandir la presencia de la escuela en el país que en enriquecer sus contenidos.

En esta segunda mitad del siglo XX se dejó atrás las ideas sobre la debilidad de la raza, la higiene y los malos hábitos que primaron hasta la década de los cuarenta. En su lugar, cuestiones como la lucha contra la pobreza, la inequidad y la inestabilidad política y económica pasaron a dominar el panorama coyuntural del país. Lo anterior descuidó el desarrollo de un campo curricular claro, organizado y sólido, olvidando la importancia de la gestión de contenidos dentro de los colegios (Neira y Rodrich, 2008, p. 40).

Esta omisión afectó a la Educación Física, cuyo rol como herramienta política daría un primer gran viraje. Documentos oficiales mostraron que la principal tendencia para estos años fue la de concebir la Educación Física como un medio para 
construir patria. Ello se vio marcado principalmente durante los gobiernos militares, en donde estas ideas cobraron mayor fuerza y atención política que durante los años democráticos. Aún así, cada gobierno mostraría diferentes matices y propuestas pese a haber concebido la Educación Física como medio importante para promover la unidad nacional. Ello se evidenciaría en el análisis de las políticas educativas ejecutadas durante los gobiernos de Manuel Odría y Juan Velasco Alvarado.

\subsection{Funciones adjudicadas a la Educación Física entre 1950 y 1979}

La idea de utilizar la educación como vía para formar a los ciudadanos y alinearlos a los intereses de una nación ha existido por siglos; y la de aprovechar la Educación Física como medio para formar individuos físicamente preparados para la lucha nacional, también ${ }^{5}$.

Durante las tres décadas comprendidas en este período, fueron más de veinte los años en los que el Perú estuvo gobernado por un gobierno militar. Por eso muchos de los textos revisados mostraron una tendencia hacia el fomento de la Educación Física y los deportes como herramienta para promover la competitividad nacional, generar la integración en el país y despertar el espíritu de lucha que se requería para defender la soberanía del país.

A diferencia del primer momento, el interés por condicionar física y moralmente el cuerpo del alumno estuvo guiado por la búsqueda de formar individuos preparados para servir a la patria. Se interpuso, antes que los beneficios individuales, los fines nacionales, y se buscó construir una predisposición y entrega voluntaria hacia los intereses y fines del país.

El primer gobierno en evidenciar esta lógica fue el de Manuel Odría, cuyo Plan de Educación Nacional constituyó un primer esfuerzo por definir objetivos, proponer métodos y organizar la gestión educativa. Enfrentando grandes brechas socioeconómicas y una creciente crisis agraria, el gobierno de Odría promovió la educación como motor de cambio para reducir dichas desigualdades, industrializar el país y alcanzar la integración nacional (Morillo, s.f.).

Bajo esta lógica, el Plan de Educación Nacional de Manuel Odría advertía que

En ningún momento debe olvidarse que es función principalísima de la educación el preparar a los educandos para que puedan servir a la colectividad con profundo sentido social, y a la Patria con claro concepto de los intereses permanentes del país [...]. Por eso, la escuela y el colegio prestarán preferente atención a la formación de la personalidad del educando en función de sus deberes sociales (Ministerio de Educación Pública, 1950, p. 30).

\footnotetext{
5 Históricamente, si no ha sido por la concepción griega de conservar un cuerpo sano para una mente sana, la función de la Educación Física ha tenido, como en la Roma antigua, fines militares.
} 
Un primer elemento característico de los textos de la época es la búsqueda de la integración y la unificación del país. La unidad y la integración nacionales fueron de la mano con el interés del gobierno por generar una identidad peruana. A diferencia de la primera mitad del siglo, esto implicó la aceptación de la raza y su autenticidad como punto de partida para fomentar la emoción social y ese peruanismo del que se jactó el Plan de Educación Nacional.

Solo con un sentido de identidad fuerte es que el gobierno ambicionó poner a la población al servicio de la comunidad y del país. El mismo plan menciona esta tarea como el fin último e incluso sagrado para la sociedad. El discurso transmite un agrandamiento de la patria, a la cual se le consideró alma de la escuela y principal rectora. También expresa de manera tácita la visión y los intereses de un gobierno militar, afirmando abiertamente que no se aprende para saber, sino para servir a la patria.

En este contexto, la Educación Física cobró importancia por su orientación a la acción, en busca del desarrollo de una actitud pujante para "... actuar en forma inmediata y feliz con demostraciones de responsabilidad, de amar al prójimo y de devoción patriótica» (Ministerio de Educación Pública, 1950, p. 14). El plan fue claro al señalar que «La finalidad por alcanzar es crear el hábito de hacer, de realizar la tarea, de producir, que va formando en la persona espíritu de lucha y fe en la acción, y luego afirmar la personalidad y tonificar el carácter en los dominios de la investigación, de la conducta social y del cumplimiento de las leyes» (Ministerio de Educación Pública, 1950, p. 14).

No es casual la alusión a "tonificar el carácter» en el texto; especialmente, si consideramos el concepto de tonificación como término que alude al fortalecimiento del organismo físico. El énfasis a la patria es utilizado como motivación para difundir un sentido de la acción en los peruanos. La Educación Física inculcaría a los niños el espíritu de acción y los adoctrinaría con los principios del militarismo patriótico.

Más allá de los límites de la escuela, el Plan de Educación Nacional de Odría buscó despertar el espíritu de acción y el patriotismo a través del deporte como actividad recreativa: «... la recreación no constituya un simple pasatiempo, sino que [...] estimule el optimismo, la fe y el espíritu de lucha [...] Es necesario limitar el individualismo que origina, a veces, sentimientos egoístas" (Ministerio de Educación Pública, 1950, p. 16). Llama la atención la forma negativa con la que se describe el individualismo como origen del egoísmo. Así, el texto justifica sus intenciones de promover objetivos colectivos.

La Educación Física, dentro de la idea de recreación, se propuso como una actividad sana y provechosa. Internamente, constituyó sobre todo una herramienta de control social que ocupó el tiempo libre de los educandos en la consecución de ciertos objetivos ambicionados por el Estado: el cooperativismo por encima del individualismo para el cumplimiento de altos fines. 
Tanto la Educación Física como el concepto de recreación perduraron en el tiempo como revitalizadores del nacionalismo y del espíritu de acción a lo largo de los distintos gobiernos militares. Prueba de ello se encontró al analizar las políticas promulgadas durante la reforma del Gobierno Revolucionario de las Fuerzas Armadas (GRFA), que fue concebida como parte importante de un paquete de reformas estructurales que buscaban sentar las bases de una nueva sociedad (Rojas, 2019). A diferencia de la visión de integración nacional de Odría, que miraba la educación como el motor para reducir las desigualdades y facilitar la industrialización del país, la reforma educativa fue solo una parte de los cambios estructurales profundos que el GRFA buscó hacer para atenuar las desigualdades y afianzar la soberanía peruana frente al dominio extranjero (Rojas, 2019).

En este contexto, la reforma educativa que impulsó Velasco apostó por una concepción tripartita de la recreación, la Educación Física y el deporte al servicio de los grandes objetivos de su revolución.

El GRFA constituyó el Instituto Nacional de Recreación, Educación Física y Deporte (INRED); quizás, dejándose influenciar por las políticas de Fidel Castro en Cuba, quien había fundado el Instituto de Deportes, Educación Física y Recreación (INDER) en $1961^{6}$. Desde el INRED, el apoyo y las reformas hacia la Educación Física se sostuvieron sobre el supuesto de que «...todas sus acciones resultan medios efectivos y reales de [...] recuperar nuestro amor profundo por la patria y a generar una entrega incondicional al servicio de su progreso, justicia social, integridad y soberanía» (Gobierno del Perú, 1972, p. 29). La Educación Física fue concebida GRFA como un vehículo para generar cohesión, compromiso y cooperación nacional. Sin embargo, a diferencia del gobierno de Odría, apeló a los ideales como el amor, la justicia social, el progreso y la soberanía en lugar de divinizar la patria para generar devoción.

Tanto en el gobierno de Odría como en el de Velasco se sobrepusieron los intereses nacionales del gobierno a los beneficios individuales de los estudiantes. La Educación Física fue vista como factor de movilidad social y de acondicionamiento físico para el trabajo que transformaría estructuralmente la sociedad y reafirmaría nuestra independencia: «Un hombre sano y fuerte para un nuevo Perú» (Gobierno del Perú, 1972, p. 92).

Complementariamente, la Educación Física en este contexto fue vista como formadora de cuerpos sanos, fuertes y preparados para la realización de trabajo

\footnotetext{
6 El INDER se fundó en 1961, cuando el gobierno de Fidel Castro dictó medidas en favor de mejorar la situación existente respecto a la práctica del deporte en Cuba. Bajo la consigna de «El deporte, derecho del pueblo», la promoción del deporte se convirtió en un principio de la revolución en Cuba.
} 
industrial, aunque sin perder de vista su enfoque corporatista en favor de los intereses del gobierno y del país.

Odría destacó en sus políticas los beneficios de la Educación Física en la conservación de la salud, el robustecimiento del vigor corporal y la recreación. Asimismo, la concibió como una forma de capacitar al alumno para el trabajo futuro, destinado a aumentar el potencial espiritual y material del país (Ministerio de Educación Pública, 1950, 1952). Por su parte, el gobierno de Velasco reconoció el valor de la recreación, la Educación Física y el deporte como campos íntimamente vinculados con la política de desarrollo económico y social del país (Ministerio de Educación, 1973, pp. 3-5).

En ambos casos, los gobiernos militares fueron más allá de la escuela para desarrollar y promover la recreación saludable a través del deporte, no siendo suficiente el desarrollo y la implementación de la Educación Física en los colegios. Los gobiernos buscaron transformar las prácticas extracurriculares, inclusive, con miras a generar individuos con un cuerpo, mentalidad y hábitos consistentes a favor de impulsar el trabajo duro y la mejora económica del país.

\subsection{Un periodo diferente}

Una diferencia respecto de las tendencias identificadas como funciones de la Educación Física durante los gobiernos militares se presentó con el análisis de documentos pertenecientes a los pocos ańos democráticos que hubo entre 1950 y 1979. En este período, el papel de la Educación Física pareció perder importancia.

Durante la presidencia de Manuel Prado Ugarteche, tanto la Educación Física como la Educación Militar fueron concebidas como actividades extracurriculares con una carga de dos horas semanales, sosteniendo que la educación debía «... procurar el adecuado desarrollo de un cuerpo sano y el equilibrado desenvolvimiento de todos aquellos atributos de la personalidad y del carácter que enriquecen su cultivo espiritual, afirman la dignidad del hombre y favorecen la consecución de sus fines inmanentes y trascendentales» (Ministerio de Educación Pública, 1969, p. 6). Aquí, la importancia atribuida a la Educación Física radicó en el desarrollo individual y el aspecto psicomotor de este proceso.

\subsection{Alcances en la práctica entre 1950 y 1979}

Cuando se lanzó el Plan de Educación Nacional (Ministerio de Educación Pública, 1950), el documento determinó la proporción de tiempo con la que cada área de estudio contaría en la escuela secundaria. Dividiendo las asignaturas en cuatro grandes áreas — letras, ciencias, trabajo manual y esfuerzo físico-, el documento asignó para los dos primeros ańos de secundaria 12,1\% del total de horas lectivas 
al área de esfuerzo físico; 9,1\% al área de trabajo manual; 27,3\% al área de ciencias, y $51,5 \%$ a letras.

Si bien es cierto que el rubro de esfuerzo físico recibió un porcentaje de tiempo bastante menor que el de letras o ciencias, su permanencia a lo largo de los cinco ańos de secundaria con la misma carga horaria muestra la relevancia de su enseñanza y práctica constante. Además, hay que tener en cuenta que las áreas de letras y ciencias incluyeron más de un curso, mientras que el área de esfuerzo físico estuvo comprendida solo por Educación Física. Visto así, la distribución horaria por curso resultaría similar a la del primer momento.

Documentos de mayor especificidad, como el Reglamento de Educación Secundaria (Ministerio de Educación Pública, 1950), establecieron para el curso de Educación Física un mínimo de dos y un máximo de seis horas semanales para los diferentes niveles educativos.

Por otra parte, observaciones en aula permiten hacerse una idea sobre el autoritarismo y la fuerte disciplina que rigió el ambiente escolar en aquel momento. Estas describen los constantes llamados al orden y a la formación en los que debían participar los niños: «El proceso de 'acercamiento al orden' dura un buen rato; los gritos de los maestros se suceden con rapidez, otros profesores recorren las filas tratando de imponer el orden...». Asimismo, narran cómo «... muchos chicos se cansan de estar ordenados, de estar tomando 'distancia' y pasando indistintamente de 'atención’ a 'descanso' y viceversa» (Ballón, Riofrío y Roncagliolo, 1974, p. 1).

Del mismo modo, entrevistas a maestros plasman la lógica usada para justificar el alto grado de rigidez dentro de la escuela: el ideal del «hombre de bien», recto, disciplinado y obediente, ante todo. Al hablar sobre la fuerte represión hacia los alumnos, un maestro declaró que «los nińos vienen a la escuela a aprender, a hacerse hombres de bien; la formación debe tranquilizarlos y si no se tranquilizan por las buenas hay que hacerlo por las malas... Es por el bien de ellos» (Ballón, Riofrío y Roncagliolo, 1974, p. 3).

En el ámbito institucional, en la década de 1950 se creó una Sección Técnica para comprender y manejar cursos como el de Educación Física dentro de las escuelas. Fueron responsabilidades de esta sección el preparar el desarrollo metódico y el control de la cultura física en las escuelas, formular los planes y las actividades deportivas anuales en los colegios, controlar y supervisar el trabajo de los profesores de Educación Física, velar por la buena conservación del material y la infraestructura deportiva, monitorear el progreso físico de los alumnos y fomentar la participación de los estudiantes en competiciones (Ministerio de Educación Pública, 1950).

Además, durante la misma década se incluyeron condecoraciones para alumnos destacados en actividades deportivas, además de las académicas. Durante el gobierno de Odría, el Decreto de Ley 11193 exigió la creación y reglamentación de 
los laureles deportivos del Perú para deportistas peruanos destacados, que pretendió fomentar la competitividad en los alumnos mediante el deporte en las escuelas y fuera de ellas (Ministerio de Educación Pública, 1950).

A diferencia de lo visto entre 1900 y 1949, las décadas de 1950 a 1980 fueron el escenario de una delimitación de contenidos en el curso de Educación Física. Las cuestiones de la higiene como contenido correspondiente a esta asignatura terminaron por desaparecer. Los conceptos de salud y alimentación quedaron definitivamente separados de la Educación Física y se desarrollaron en cursos separados como Educación Familiar y Educación Cívica (Cajas, 1957).

En paralelo, los procesos de matrícula fueron simplificados y dejaron de exigir la presentación de elementos como la indumentaria específica para el día y la noche, tal como sucedía desde en las primeras décadas del siglo XX.

Finalmente, las décadas de 1950 a 1980 mostraron expectativa por fomentar el deporte y la competitividad internacional del país. Primero, a través la creación de secciones técnicas para promover la Educación Física y los deportes competitivos en las escuelas en 1950, el aumento de los reconocimientos a alumnos destacados en la Educación Física y los deportes el mismo año, y la creación de los laureles deportivos en 1959.

Luego, a través de la creación del INRED — antepasado del Instituto Peruano del Deporte (IPD) — , que implicó un plan a largo plazo orientado a mejorar la infraestructura para la Educación Física entre un 136\% — para el aumento y mejoramiento de canchas de fútbol — y un 4739\% — para la construcción de gimnasios. También aspiró a aumentar la cifra de profesores titulados en 3500, la de los técnicos auxiliares en 1570, la de entrenadores en 1200, la de masajistas en 630, la de médicos especializados en 289, y la de kinesiólogos en 250 (Gobierno del Perú, 1972).

\section{1980-1990: EdUCACIÓN FísICA COMO PARTE DEL DESARROLLO INDIVIDUAL}

Tras el fin de la segunda etapa del GRFA llegaría en 1980 la democracia al Perú. Para entonces, el país sufría un severo problema social que en el ámbito educativo se tradujo en la disminución de la calidad, un problema de cobertura ${ }^{7}$ y crecientes

\footnotetext{
Para 1988, el texto del Ministerio de Educación Politica educativa para la democracia, la conciencia histórica y el desarrollo dio como diagnóstico general el déficit del sistema educativo, proponiéndose universalizar y democratizar el acceso y la permanencia en de los peruanos en un sistema educativo gratuito (Cabanillas, 1988). En busca de lograr este objetivo, el mismo documento reconoció la necesidad de «afianzar el proceso de descentralización educativa, mediante la participación de los municipios en la vigilancia del normal funcionamiento de la educación primaria; y en programas de alfabetización, construcción escolar, cultura, recreación y deportes, de acuerdo con lo previsto por la Constitución del Estado, la Ley de Educación y la Ley Orgánica de Municipalidades» (Cabanillas,
} 
tensiones entre el Estado y los maestros. Por si fuera poco, Sendero Luminoso empezaba a infiltrarse en las escuelas, aprovechándose del gran ausentismo del Estado en los colegios del sector público.

Muchas de las reformas implementadas en la década de 1980 implicaron desechar las políticas establecidas por el gobierno militar. En el sector educativo, la nueva Ley General de Educación 23384 (Gobierno del Perú, 1982) descartó los cambios hechos por la Reforma Educativa de 1972. El Decreto Legislativo 135 renombró el INRED como el Instituto Peruano del Deporte (IPD) y lo dotó de una nueva dirigencia: el Comité Nacional del Deporte. Finalmente, la Ley General del Deporte fue sustituida por la Ley Orgánica del Deporte Nacional en 1985.

Si bien las legislaciones señaladas tuvieron como propósito la promoción y el desarrollo del deporte, poco fue lo que se hizo en esta materia; menos aún, lo que se hizo en las escuelas con la Educación Física.

Ante una bancarrota estatal, altísimos niveles de corrupción, mucha violencia interna y un sindicato de maestros que se fortalecía y presionaba cada vez más al Estado, los problemas curriculares de la educación peruana, sus objetivos y contenidos, terminaron por desaparecer del primer plano.

El rol social y político de la Educación Física se perdió de vista. Más allá del valor de dicha materia por los beneficios que ofrecía al educando mismo, la materia perdió la función sociopolítica que había mantenido en los períodos anteriores. La democracia parecía prescindir de la Educación Física como vehículo de soporte para los proyectos políticos del Estado.

\subsection{La nueva visión de la Educación Física}

En la Ley General de Educación 23384 (Gobierno del Perú, 1982) se describe la educación peruana como garante del desarrollo integral del individuo y la sociedad. Bajo dicha lógica, los contenidos plasmados y desarrollados dentro del sistema educativo peruano preparaban al individuo para actuar dentro de la sociedad en pacífica coexistencia.

Pese a mantener tanto la Educación Premilitar como la Educación Física como cursos obligatorios, la ley delegó la Educación Premilitar al Ministerio de Guerra y asoció la Educación Física con el desarrollo psicobiológico y la promoción de la integración del educando a la comunidad organizada (Gobierno del Perú, 1982, p. 3). Esto deslindó la preparación premilitar como responsabilidad del Ministerio de Educación y desmilitarizó los contenidos de la Educación Física, dejando de lado los argumentos nacionalistas de otros momentos a cambio de argumentos

1988, p. 79). Esta información oficial da cuenta de la falta de cobertura y calidad que enfrentaba, en ese entonces, el sistema educativo peruano. 
individualistas y psicomotrices, respaldados por la ciencia de la educación y ya no por ideologías.

Otros documentos, como la Ley General de Educación 23384 o el Plan del Sector Educación 1986-1987, mantuvieron un discurso similar. Aunque anunciaron la reestructuración institucional y actualización curricular, calificaron la Educación Física como un "servicio educativo complementario» y de segundo orden (Ministerio de Educación, 1982, 1986).

Más aún, políticas adicionales (Cabanillas, 1988) afirmaron que la Educación Física hasta ese momento no cumplía el papel que le correspondía; y que los propósitos hasta entonces adjudicados a la asignatura no eran los correctos. Esto sugiere un rechazo a las concepciones con las que anteriormente fue vista la Educación Física. También apostaron por una reconceptualización y reorganización de la materia que partiera de un enfoque psicobiológico: para conocer el cuerpo de uno mismo y generar en él sensaciones de plenitud y satisfacción, en lugar de sobrexigirlo en busca de objetivos más ambiciosos.

La Educación Física se mantuvo dentro de los currículos en este período para velar por un desarrollo psicomotriz del alumno, que se consideraba imprescindible para el desarrollo del intelecto. El fin último, sin embargo, fue el de preparar el cuerpo para un mejor aprendizaje y desempeño académico.

\subsection{Funciones adjudicadas a la educación física entre 1980 y 1990}

Aunque los objetivos de generar unidad e identificación nacionales a través de la educación se mantuvieron vigentes en la década de 1980, los cursos previstos para cumplir dichos propósitos en la práctica fueron distintos de los de períodos anteriores. La integración e identidad nacionales se buscaron a través de la Educación Cívica y no a través de la Educación Física. Esto se enmarcó en un contexto que buscó afirmar la democracia y romper con los rezagos dejados por el último gobierno militar (Gobierno del Perú, 1982).

La democracia propuso "Alcanzar un alto nivel cultural, humanista y científico, como un valor en sí y como indispensable instrumento de progreso» (Gobierno del Perú, 1982, p. 1), y planteó el resurgimiento de los contenidos académicos como pilares del progreso educativo y del afianzamiento de la conciencia histórica de los estudiantes para fomentar la cooperación, el desarrollo y la integración (Cabanillas, 1988, p. 59).

Si anteriormente se procuró alcanzar la unión y el progreso usando métodos militarizados, el fomento a la competitividad entre naciones y el cultivo de un espíritu de lucha en la Educación Física, este documento marcó un giro al proponer la restauración cultural como cimiento para la integración nacional. 
En este momento histórico apareció por primera vez el concepto de una educación integral, comprendida por las dimensiones corporal y espiritual del individuo. Desde este enfoque, tanto los contenidos academicistas como los aprendizajes prácticos, incluyendo la Educación Física, resultaban componentes fundamentales de todo proceso educativo; y aunque la materia formó parte de dicha educación integral, hubo una clara intención por reforzar otros contenidos.

Aspectos como la higiene o los valores cívico-patrióticos continuaron vigentes durante la década de 1980, mas no mantuvieron una relación medios-fines con la práctica del deporte o de la Educación Física. El curso tuvo poco que aportar al proyecto de construcción de democracia y la formación de una identidad nacional pacífica. Para los gobiernos democráticos, la construcción de una sociedad unida y pacífica debía partir del cultivo de valores éticos y cívicos, así como de nuestro pasado común. Por eso, cursos como la Historia del Perú o la Educación Cívica tomaron el lugar de la Educación Física como asignaturas estratégicas en busca de la construcción nacional.

\subsection{Alcances en la práctica entre 1980 y 1990}

El nuevo enfoque educativo adoptado por la democracia se hizo notar en una diversificación de los cursos en el currículo escolar. En el Plan de Estudios de Educación Secundaria para el Área de Ciencias y Humanidades y para las Áreas Técnicas de 1983, se insertaron materias como Psicología, Economía Política, Filosofía y Lógica y Educación Cívica (Ministerio de Educación, 1983). La Educación Física constituyó entre el cinco y el seis por ciento del total de las horas lectivas en este plan, recibiendo una carga de dos horas semanales en cada ańo lectivo.

Cursos con carga similar o menor fueron Orientación y Bienestar del Educando, Educación Artística, Educación Cívica y Educación Religiosa. En cambio, asignaturas como la de Lenguaje y Literatura, Matemática, Ciencias Naturales, Formación Laboral e Historia del Perú recibieron entre el once y el quince por ciento del total de horas lectivas. Una distribución horaria así puede parecer normal o lógica desde un punto de vista actual. Sin embargo, es importante recordar que, décadas atrás, el curso había recibido mayor o igual peso que asignaturas como Historia del Perú, Lenguaje y Ciencias Naturales. Es este gran giro el que corresponde resaltar.

Durante la década de 1980, diversos documentos promovieron el deporte y la recreación, pero, más que como parte de un programa educativo integral, como forma de utilizar el tiempo libre de manera productiva (Ministerio de Educación, 1986).

Ahora bien, algunos documentos oficiales fueron realistas al describir algunos de los problemas fundamentales que enfrentaron tanto la Educación Física como los deportes y la recreación en aquel entonces: la inexistencia de un sistema educativo 
garante de la coherencia y de sus contenidos, y la insuficiente infraestructura para la práctica de la Educación Física y el deporte en los centros educativos (Ministerio de Educación, 1986). Asimismo, expusieron la paupérrima situación del deporte en las escuelas de manera sintética:

Muy agradable sería que cada cinco secciones en el Perú pudieran disponer de una pelota. Para ello deberíamos gastar S/. 3602000 000. Mejor sería no mencionar la cantidad que requeriríamos para proporcionar infraestructura o equipamiento deportivo, cuyo valor pedagógico todos aprecian plenamente (Pango, 1985, p. 2).

Pese a haber mostrado una intencionalidad por reforzar la infraestructura y fomentar y la práctica de la Educación Física y el deporte en las áreas menos favorecidas del país (Pango, 1985, p. 57), la principal limitante para los gobiernos de la década de 1980 fue la falta de presupuesto. La Educación Física no estuvo incluida entre las acciones prioritarias: la expansión de la educación, la lucha contra el analfabetismo, el desarrollo de una educación tecnológica y ocupacional, la profesionalización y formación magisterial, la expansión de la educación en áreas rurales y urbano marginales, la descentralización y desburocratización administrativa del sector educativo y el mejoramiento de la infraestructura educativa (Pango, 1985, pp. 79-81).

Conociendo el nivel de urgencia y la gran importancia de dichas prioridades para el gobierno y la sociedad peruana, y comprendiendo que, desde este nuevo contexto, la Educación Física no se prestaba como medio para la acción política, no resulta extraño que la asignatura haya quedado olvidada en el currículo nacional.

De hecho, artículos mediáticos afirmaron incluso que el curso fue eliminado del currículo nacional en 1985 durante la gestión de Mercedes Cabanillas en el Ministerio de Educación (El Comercio, 31 de agosto de 2008). Sin embargo, Cabanillas (2 de agosto de 2013) califica la falsa información circulando por los medios como infundios políticos y asegura no solo no haber eliminado la Educación Física del currículo nacional, sino haber incrementado las horas lectivas a todos los programas educativos (primaria, secundaria y educación técnica):

[...] nosotros auspiciamos y llevamos a la práctica una reforma curricular auténtica en base a una, a un mayor tiempo educativo. Dispusimos, y así se hizo, hasta que terminó el período en el año 90, eh...incorporar los días sábados a las actividades curriculares [...] Por eso es que de 35 horas semanales usuales pasamos a 42 [...] Entonces nosotros... si a la Educación Física a veces con una hora, a veces con dos... nosotros le pusimos tres (Cabanillas, 2 de agosto de 2013).

Las declaraciones de Cabanillas encontraron sustento en las Normas para la Organización y Desarrollo del Año Escolar 1989 - Educación Primaria y Educación Secundaria (Ministerio de Educación, 1989), documento que implementó 
planes piloto y que otorgó a la Educación Física tres horas lectivas por semana. En concordancia con el proceso de democratización del país y los lineamientos planteados en otros documentos, la normativa le dio una mayor importancia al curso de Educación Cívica, pero añadió horas a la Educación Física también. Junto a estas medidas, el mantenimiento de la Educación Premilitar y su ya mencionada delegación al Ministerio de Guerra mostraron coherencia con el enfoque democrático e integral con el que se trabajó la Educación en este tiempo, delimitando y distinguiendo estos temas y sus respectivas funciones con mayor claridad y cuidado.

Pese a estas iniciativas, la Educación Física no dejó de atravesar limitaciones como en años anteriores. En este caso, Cabanillas lamentó la ausencia de profesores de Educación Física titulados: «... en aquella época, te hablo del ocho siete, ocho nueve, no había oferta adecuada de profesores de Educación Física [...] O sea, tenían las horas y había déficit» (Cabanillas, 2 de agosto 2013). Para completar el número de docentes requeridos, se recurrió a técnicos deportivos, sin conocimientos de docencia.

\section{CONCLUSIONES Y REFLEXIONES FINALES}

La Educación Física se insertó en la escuela peruana a principios del siglo XX para enfrentar problemáticas de la sociedad peruana en aquel entonces. Desde una propuesta positivista o civilizadora, se trató de «fortalecer a la raza peruana y de combatir los malos hábitos» para lograr la industrialización y la modernización del país.

Luego de ser derrotados por el ejército chileno en la Guerra del Pacífico, la sociedad peruana no solo fue vista como débil por las élites, sino que para muchos quedó inmersa en malos hábitos como la holgazanería, el juego y el alcohol. En ese contexto - y a partir del ejemplo de las experiencias europea y estadounidense-, la Educación Física se incluyó dentro de los currículos para formar individuos burgueses saludables y vigorosos, con una buena disciplina y una correcta moral.

Es por eso por lo que, entre 1900 y 1949, la Educación Física fue una materia importante en el proceso de industrialización y modernización del país; y los gobiernos de esos años evidenciaron un alto interés por institucionalizar y desarrollar entidades que gestionaran y promovieran la Educación Física en las escuelas.

Ese alto interés se vio reflejado en los currículos nacionales, en donde la asignatura se convirtió rápidamente en un complemento de la Educación Premilitar y recibió más horas semanales que cursos como Geografía, Historia Antigua, Historia del Perú o Idiomas. La materia abarcó una gran amplitud de temas, desde cuestiones de limpieza e higiene hasta ejercicios aplicados al trabajo futuro; se trató, en este período, de una materia que fue más allá de los ejercicios físicos en sí mismos. 
Para la segunda mitad del siglo, entre 1950 y 1979, la idea de que fortalecer la raza y eliminar los malos hábitos era posible a través de la Educación Física perdió peso. En el contexto de más de veinte años gobernados por fuerzas militares, la función de la Educación Física quedó íntimamente relacionada a la Educación Premilitar y a la generación de una identidad nacional y de un espíritu de lucha.

Se utilizó la Educación Física como un medio para construir patria. Junto con la Educación Premilitar, la Educación Física fue orientada a promover que los estudiantes se identificaran con los intereses nacionales, y a generar una predisposición y entrega voluntaria hacia lo nacional. La gran funcionalidad que la Educación Física y el deporte tuvieron para los gobiernos militares traspasó los límites de la escuela, llegando a promoverse por el Ministerio de Educación también fuera del ámbito escolar.

Además de mostrar un gran cambio en la funcionalidad social y política de la Educación Física a partir de la década de 1950, los años 1950 a 1979 ponen en evidencia la correlación existente entre el tipo de gobierno y su aproximación al curso de Educación Física, y la susceptibilidad de los contenidos curriculares a los intereses políticos y sociales del gobierno. Esto, al comprobarse el distinto manejo de la Educación Física, el deporte y la recreación que hubo durante los gobiernos militares y los democráticos de este período.

Finalmente, aunque la democracia que llegó en 1980 también persiguió la unidad nacional y el desarrollo del país, sus medios no fueron los mismos ni estuvieron sustentados sobre las mismas bases. Queriendo alejarse de lo heredado por los años de militarismo en el país, la democracia buscó generar conciencia nacional y ciudadanía a través de la Educación Cívica, la Ética y la Historia del Perú, evocando a un pasado en común y no al espíritu de lucha o de servicio, como se hacía anteriormente con la Educación Física.

Los gobiernos democráticos que llegaron a partir de 1980 rechazaron las antiguas aproximaciones a la Educación Física. Sus políticas educativas curriculares desvincularon la Educación Física tanto de contenidos sobre limpieza e higiene como de la Educación Premilitar, y buscaron reconceptualizarla en función al desarrollo del individuo, en lugar de orientarla en función de intereses sociopolíticos.

Vista desde este nuevo enfoque, la asignatura vio disminuidas sus horas lectivas y pasó a considerarse un curso complementario o una actividad de tiempo libre. Por si fuera poco, las crecientes limitaciones en el presupuesto, la infraestructura, el material, el personal docente titulado, y la inevitable necesidad de priorizar las políticas educativas colocaron la regulación de la Educación Física al final de la cola.

Desde entonces, las recientes políticas para incentivar el desarrollo de la Educación Física, la recreación y el deporte parecen reafirmar los hallazgos de esta investigación. Pese a existir el Plan Nacional del Deporte 2011-2030 (IPD, 2011), 
que marca los lineamientos para el desarrollo del deporte a largo plazo, pocos son los avances en materia de Educación Física.

En el 2014, el Plan Nacional de Fortalecimiento de la Educación Física y el Deporte Escolar en las Instituciones Educativas de Primaria y Secundaria de la Educación Básica Regular (Ministerio de Educación, 2014) contempló el incremento de horas lectivas para la Educación Física en los colegios ${ }^{8}$, la contratación de docentes de esta materia; la capacitación a los profesores; y la habilitación de mejor infraestructura y la compra de materiales. Al plan se le asignó primero un presupuesto de 70 millones de nuevos soles ${ }^{9}$, que luego ascendió a 150 millones en 2015. Pese a estos esfuerzos, los objetivos de este plan solo se han desplegado en una pequeña cantidad de colegios a nivel nacional.

Recién en 2016, cinco años después de publicado el Plan Nacional del Deporte 2011-2030 se creó la Dirección de Educación Física en el Ministerio de Educación y el programa curricular de Educación Física para la escuela primaria. Sin embargo, hasta 2018 el Ministerio de Educación admitía dificultades para medir el progreso físico de los alumnos en la escuela, además de una severa escasez de personal docente: para contar con un profesor de Educación Física por escuela, hacen falta 41000 profesionales (Integración, 2018).

En la actualidad, la mejor forma encontrada por el Estado para promover el deporte en la escuela ha sido la realización de los Juegos Deportivos Nacionales Escolares. Sin embargo, la evidencia muestra que menos del 1\% del alumnado participa en este evento de manera constante (Integración, 2018).

Es cierto que el Estado cuenta con iniciativas como el Programa de Apoyo al Deportista (PAD), que apoya económicamente a deportistas de alto rendimiento y les ofrece alojamiento e infraestructura de calidad a través de Centros de Alto Rendimiento (CAR). No obstante, estas iniciativas no solo apoyan solo al 10\% del total de federaciones deportivas en el país, sino que no desarrollan el deporte desde la infancia.

Cuba, el país con mejores resultados deportivos de Iberoamérica, capta a sus deportistas de élite desde la Educación Física escolar, a través de un programa fundado en 1961: el mismo año que Fidel Castro fundó el INDER que inspiró a Velasco para fundar el INRED. Actualmente, el Perú capta deportistas de alto rendimiento a través de talleres deportivos electivos dirigidos a niños y adolescentes.

\footnotetext{
8 La propuesta pedagógica « $2+3$ » busca añadir a las dos horas regulares que tiene el curso de Educación Física tres horas de jornada ampliada, destinadas a fortalecer la expresividad motriz; fomentar un estilo de vida activa y saludable, y permitir el desarrollo sociomotor y deportivo de los estudiantes. Esto permitiría también identificar talentos en una etapa escolar y trabajar en la especialización deportiva y la participación en competencias de alta competencia.

9 Presupuesto del sector público para el año fiscal 2014.
} 
Esto limita el semillero deportivo del país, ya que la captación está supeditada a que los padres inscriban a sus hijos en dichas actividades (Integración, 2018).

Queda aún mucho por investigar en el terreno de la Educación Física y el deporte en el país. Esta revisión histórica solo pretende ser un punto de partida para trabajar estos temas con una visión de largo plazo, menos improvisada y cortoplacista. Los intentos de orientar el deporte en diferentes direcciones pueden deben ser vistos como proyectos políticos de Nación, en la medida que modelan formas de ciudadanía y tipos de vínculos entre personas en el territorio nacional: Refuerzan identidades y tienen el potencial de contribuir al progreso del país ${ }^{10}$. En el Perú, esta es una agenda inconclusa.

\section{REFERENCIAS}

Abrams, P. (1983). Historical Sociology. Nueva York: Cornell University Press.

Augustin, J. (1934). La educación física escolar. Lima: Impr. Minerva.

Ballón, E., Riofrío, G. y Roncagliolo, R. (1974). Cuadernos DESCO: El maestro en la escuela hoy. Lima: DESCO.

Beleván García, C. (1962). Notas para la historia de la educación física y el deporte: apuntes del curso dictado durante los años académicos de 1960-1961. Lima: Instituto Nacional de Educación Física.

Cabanillas, M. (1988). Política educativa para la democracia, la conciencia histórica y el desarrollo. Lima: Ministerio de Educación.

Cabanillas, M. (2013). Entrevista personal. Lima: 2/8/2013.

Cajas, A. (1957). Historia de la educación física en el Perú. Lima: Impr. Gil Armas.

Deustua, A. (1902). La cultura nacional. Lima: UNMSM.

Eguiguren, L. A. (1915). La holgazanería en el Perú. Lima: Impr. de E. Moreno

Elias, N. y Dunning, E. (1992). Deporte y ocio en el proceso de la civilización. México: Fondo de Cultura Económica.

Gobierno del Perú (1901). Ley Orgánica de Instrucción. Lima: Gobierno del Perú.

Gobierno del Perú (1906). Plan de educación. Lima: Gobierno del Perú.

Gobierno del Perú (1941). Ley Orgánica de Educación Pública. Lima: Gobierno del Perú.

Gobierno del Perú (1972). Interpretación y ayudas visuales de la Ley General de Recreación, Educación Física y Deportes: decreto de ley n. ${ }^{\circ}$ 20555. Lima: INRED.

Gobierno del Perú (1982). Ley n. ${ }^{\circ}$ 23384. Ley General de Educación. Lima.

Goodson, I. (2000). El cambio en el currículum. Barcelona: Octaedro.

Integración (2018). «Una política deportiva de pocas medallas». Lima:Instituto de Integración.

Disponible en: http:/www.integracion.pe/una-politica-deportiva-pocas-medallas/

10 Impactan en la salud física y mental de los individuos, desarrollan patrocinios privados que inyectan capital al desarrollo del país y generan formas de entretenimiento e identidad nacionales (Integración, 2018). 
Luna, N. y Canelo, P. (31/08/2008). El Estado invierte solo S/. 3 por cada peruano para el deporte. Diario El Comercio, 31/08/2008, p. A08. Lima: El Comercio.

Martuccelli, D. y Dubet, F. (1997). En la escuela: Sociología de la experiencia escolar. Buenos Aires: Losada.

Ministerio de Educación (1974). Reforma de la educación peruana. Informe general. Lima: Imprenta Editores Tipo - Offset.

Ministerio de Educación (1983). Educación fisica: programa curricular, tercer grado, cuarto grado, quinto grado. Lima: INIDE.

Ministerio de Educación (1984). Educación Física. Programa curricular. Lima: Ministerio de Educación. Dirección General de Primaria y Secundaria.

Ministerio de Educación (1986). Plan del sector educación 1986-1987. Lima: Ministerio de Educación.

Ministerio de Educación (1989). Normas para la organización y desarrollo del año escolar 1989 - Educación Primaria y Educación Secundaria. Lima: Dirección General de Educación Primaria y Secundaria.

Ministerio de Educación (2014). Lineamientos para la ejecución del Plan Nacional de Fortalecimiento de la Educación Física y el Deporte Escolar en las Instituciones Educativas Públicas de Primaria y Secundaria de Educación Básica Regular. Lima: Ministerio de Educación.

Ministerio de Educación Pública (1937). Plan de estudios y programas para las escuelas fiscales de enseñanza primaria conforme al plan oficial. Lima: Imprenta Larrabure.

Ministerio de Educación Pública (1939). Legislación educacional 1935-1938. Compilación cronológica de leyes, decretos y resoluciones correspondientes a los años 1935 y 1938. Lima: Ministerio de Educación Pública.

Ministerio de Educación Pública (1950). Plan de educación nacional. Lima: Ministerio de Educación Pública.

Ministerio de Educación Pública (1950). Laureles deportivos del Perú. Decreto-ley de creación y reglamento. Lima: Ministerio de Educación Pública.

Ministerio de Educación Pública (1950). Reglamento de educación secundaria. Lima: Ministerio de Educación Pública.

Ministerio de Educación Pública (1952). Politica educativa del régimen. Discurso pronunciado en las cámaras legislativas por el Ministro de Educación Pública, Gral. D. Juan Mendoza Rodríguez, y respuesta a los pedidos y sugerencias de los señores representantes, con motivo de la discusión del presupuesto del ramo, los días 20 y 21 de febrero de 1952. Lima: Ministerio de Educación Pública.

Ministerio de Educación Pública (1969). Programas para la educación secundaria. Lima: Edición Oficial.

Ministerio de Instrucción (1912). Reglamento general de instrucción media especial, academias y cursos libres. Lima: Empresa tipográfica Unión Boza.

Ministerio de Instrucción (1920). Proyecto de ley orgánica de instrucción. Lima: Impr. Gloria. 
Ministerio de Instrucción (1920). Guia y reglamento general de educación física (distribución gratuita a maestros, profesores de educación física e instructores militares de los colegios de la República). Lima: Litografía e imprenta Scheuch.

Ministerio de Instrucción (1929). Dirección e Estudios y Exámenes. Plan de estudios y programas para los colegios de instrucción media. Lima: Imp. La Revista.

Morillo, E. (s.f.). Reformas educativas en el Perú del siglo XX. Revista Iberoamericana de Educación - Organización Iberoamericana de Educación. Recuperado de https:// rieoei.org/historico/deloslectores/233Morillo.PDF

Muñoz, F. (2001). Diversiones públicas en Lima: 1890-1920. La experiencia de la modernidad. Lima: Red para el Desarrollo de las Ciencias Sociales.

Neira, P. y Rodrich, H. (2008). Cambios curriculares en la secundaria 1996-2006: opiniones de exfuncionarios y docentes de escuelas públicas. Economía y Sociedad, 68, 40-49. Recuperado de http://cies.org.pe/files/economia-sociedad/E-y-S-68-PaulNeira.pdf

Oliart, P. (2011). Políticas educativas y la cultura del sistema escolar en el Perú. Lima: IEP.

Pango, G. (1985). Educación para la vida. Exposición del profesor Grover Pango Vildoso, Ministro de Educación, ante la Comisión de Educación, Ciencia y Cultura de las Cámaras de Senadores y Diputados. Lima: Imprenta del Ministerio de Educación.

Pinto, R. (1915). Ensayo sobre sistemas de educación física con aplicación al Perú: apuntes tomados de las elecciones que dicta el profesor señor Raúl Pinto, en la Escuela Normal de Varones. Lima: Imp. del Centro Editorial Beytia.

Rojas, R. (2019). Velasco, la reforma educativa y los maestros. Revista Argumentos, 13(2). Lima: Instituto de Estudios Peruanos. Recuperado de https://argumentos-historico. iep.org.pe/wp-content/uploads/2019/10/Rojas-R.-2019-Velasco-la-reforma-educativa-y-los-maestros.pdf

Soler, S. (2011). Análisis crítico del discurso de documentos de política pública en educación. Forma y Función, 24(1), 75-105. Recuperado de https://www.redalyc.org/ pdf/219/21922416005.pdf 